\title{
Heartbeat: Is biologic age best defined by exercise capacity?
}

\section{Catherine M Otto}

Currently, primary prevention of cardiovascular (CV) disease is targeted towards patients at higher risk of adverse events based on scores derived from a combination of clinical factors such as age, sex, hypertension, diabetes, smoking, cholesterol levels and other co-morbidites. The concept of biologic age as an approach to risk stratification was first proposed based on anatomic evidence of subclinical coronary artery disease in asymptomatic patients. The hypothesis that anatomic measures of vascular disease better reflect the patient's biologic, rather than chronologic, age is supported by the additive predictive value of coronary calcium scores. However, the clinical utility of vascular imaging for primary prevention remains controversial. In addition, focusing on vascular anatomy alone may not fully describe the degree of vascular disease. Little attention has been directed to physiologic measures of biologic age as predictors of $\mathrm{CV}$ outcome.

In this issue of Heart, the relationship between exercise capacity and CV outcomes was assessed in a retrospective cohort study of over 57 thousand adults (without known coronary artery disease or heart failure) who underwent exercise stress testing at a single institution over an 18 year period (see page 431). Although exercise capacity decreased with age as expected, higher exercise capacity was associated with greater survival in all age groups based on endpoints of all cause mortality at 10 years $(n=6356)$ and myocardial infarction $(n=1646)$ at 5 years follow-up (figure 1). When exercise capacity was used to define biologic age based on linear parametric models for mortality risk, variation by up to 3 decades was seen within each age decile. The authors propose that "biologic age may be a useful clinical tool for facilitating patient discussion regarding the impact of exercise capacity on long-term risk." This concept is illustrated with a graph (and equations) for expected exercise capacity and a look-up table for determining biologic age based on actual exercise capacity, stratified by age and gender. A video abstract provides a quick summary and discussion of these results.

Correspondence to Professor Catherine M Otto, Division of Cardiology, University of Washington, Seattle, WA 98195, USA; cmotto@u.washington.edu
In the accompanying editorial, Budoff (see page 415 ) notes that all the patients in this large cohort had exercise testing for clinical indications, including symptoms of chest pain $(62 \%)$, shortness of breath (9\%), or preoperative evaluation (2\%), so that the applicability of this data to asymptomatic adults is not clear. He further challenges the authors' conclusion that exercise testing is useful for risk stratification of asymptomatic adults. On the other hand, "for the symptomatic population, this is a clever, readily available test to help patients understand their CV risk and relative age, and hopefully promote better behaviours to improve exercise capacity and thus morbidity and mortality."

Exercise testing is increasingly used for evaluation of older patients with suspected heart disease but it often is challenging to determine whether an apparently reduced exercise capacity or abnormal hemodynamic response is due to normal aging or an underlying disease
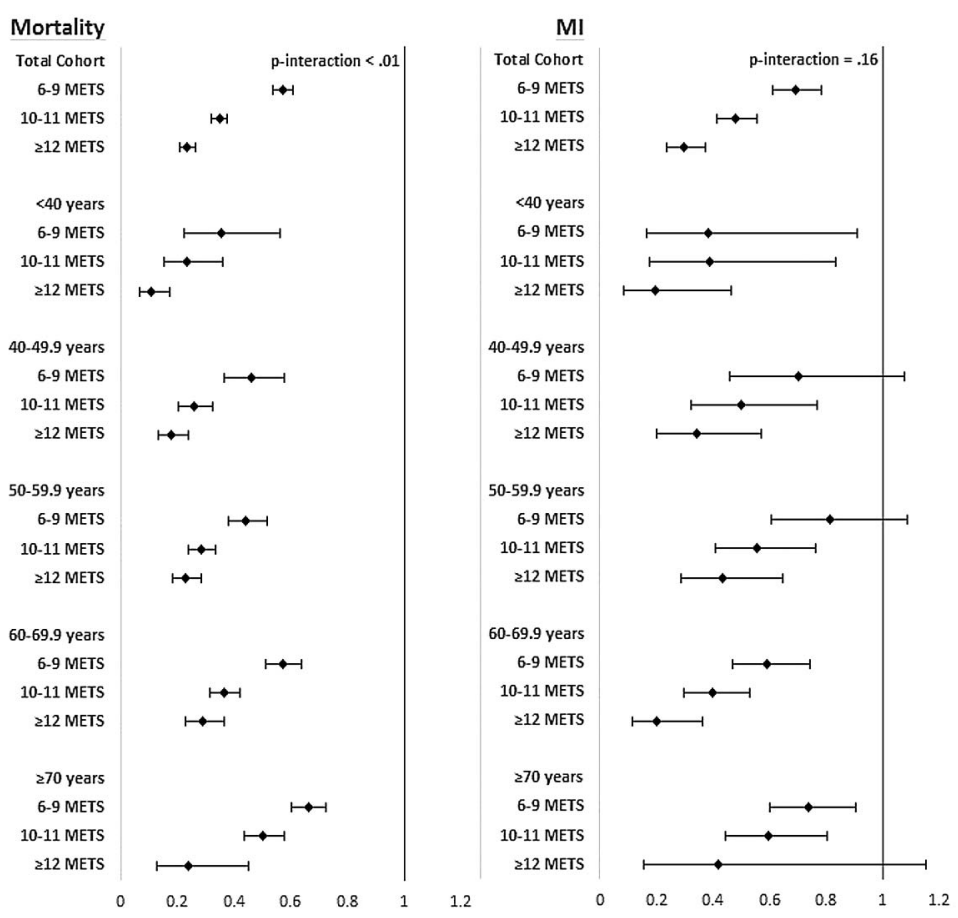

Figure 1 Adjusted hazard ratios for mortality and MI associated with increasing METS categories in the total cohort and by age groups. Per-MET HR available in online supplementary materials. $95 \% \mathrm{Cls}$ shown by error bars. P-interaction denotes significance of interaction between age and exercise capacity for each outcome. Notably, older age consistently and progressively attenuated the association between exercise capacity and mortality $\left(P_{\text {interaction }}<0.01\right)$, but not the association between exercise capacity and $\mathrm{MI}\left(\mathrm{P}_{\text {interaction }}=0.35\right)$. Models adjusted for age, gender, race, resting heart rate, resting systolic and diastolic blood pressure, history of diabetes,

hypertension, obesity, smoking, family history of $C A D$, medications for treatment of hypertension, hyperlipidaemia and COPD, and indication for stress testing. METS, metabolic equivalents; MI, myocardial infarction; $C A D$, coronary artery disease; $C O P D$, chronic obstructive pulmonary disease. 



Figure 2 Absolute changes in pulmonary and pulmonary artery wedge pressures with exercise compared with baseline: mPAP and PASP (left), PAWP and PADP (right). The changes in pulmonary and pulmonary artery wedge pressures exhibited a similar pattern at each exercise intensity between early and sustained phases. Data are presented as mean $\pm S D$. mPAP, mean pulmonary artery pressure; PASP, pulmonary artery systolic pressure; PAWP, pulmonary artery wedge pressure; PADP, pulmonary artery diastolic pressure.

exercise (figure 2). With either light or moderate exercise, PA pressures peaked after an average 3 minutes of exercise and then declined significantly after 7 minutes of sustained exercise.

This normal biphasic response to exercise is older adults likely explains the need for a "warm up" period when starting exercise; confirming what many of us know from own own experience that the first few minutes of exercise are often the most difficult. This data also suggests that measurements made after several minutes of sustained exercise might better reflect pathophysiology than measurements made within a few minutes of starting exercise. Further, we should be cautious in interpretation of an increase in pulmonary pressures with exercise because the range of responses in these normal asymptomatic individuals was quite broad with some having an increase in
PA systolic pressure of as much as 20 to $30 \mathrm{~mm} \mathrm{Hg}$. The possibility that these "normal" adults had subclinical heart failure with preserved ejection fraction cannot be definitively excluded. However, the volunteers in this study were asymptomatic and underwent both extensive clinical screening and a resting echocardiogram, so are unlikely to have significant heart disease. It is probable that the explanation for exercise elevations in pulmonary pressures in older adults is related to left ventricular diastolic dysfunction, given that echocardiographic studies show impaired diastolic relaxation is in most adults over age 50 years. The question remains whether age-related diastolic dysfunction is an inevitable "normal" consequence of aging or an abnormal modifiable disease process.

Also in this issue of Heart, Birnie and colleagues summarize the recent Consensus



Figure 3 Consensus recommendations for ICD implantation in patients diagnosed with cardiac sarcoidosis. CMR, cardiovascular magnetic resonance; ICD, implantable cardioveter defibrillator; LVEF, left ventricular ejection fraction; RV, right ventricle; VT, ventricular tachycardia (reproduced with permission from Birnie DH, Sauer WH, Bogun F, et al. HRS expert consensus statement on the diagnosis and management of arrhythmias associated with cardiac sarcoidosis. Heart Rhythm 2014;11:1305-23).

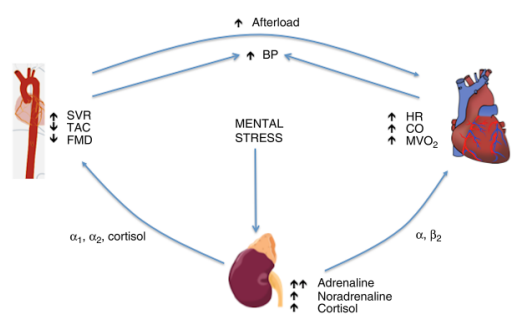

Figure 4 The acute effects of mental stress. Levels of adrenaline increase more than noradrenaline, in contrast to exercise. This leads to increased cardiac output and increased systemic vascular resistance (SVR), increasing blood pressure (BP) and cardiac afterload. $\alpha$, $\alpha$-adrenergic receptor; $\beta$, $\beta$-adrenergic receptor; $\mathrm{CO}$, cardiac output; FMD, flow-mediated dilatation; $\mathrm{HR}$, heart rate; $\mathrm{MVO}_{2}$, myocardial oxygen demand; TAC, total arterial compliance.

Statement on the Diagnosis and Management of Arrhythmias Associated with Cardiac Sarcoidosis (see page 411). The criteria for diagnosis of cardiac sarcoidosis are presented in a table and an algorithm is provided for decision making about placement of an implanted cardioverter-defibrillator (ICD) (figure 3).

The Education in Heart article in this issue discusses mental stress-induced myocardial ischaemia (see page 472). The learning objectives for this article are to understand normal coronary physiology, the cardiovascular responses to mental and exercise stress, and the relationship between mental stress, myocardial ischemia and adverse cardiovascular events (figure 4).

The Image Challenge feature in Heart has been a great success! We receive a continuous stream of high quality submissions, many more than we can publish in the print issues of the journal. Thus, starting with this issue, we will be publishing both one case in the print journal (see page 481) and an additional case online with the online case typically emphasizing diagnostic aspects of video images. Each Image Challenge will continue to be formatted as a board-review type multiple choice question with a detailed explanation of the correct answer to optimize the educational value. In addition, all of the previously published Image Challenges (over 60 to date) can be viewed on the Heart website under the "Topic Collections" tab which is a fun and easy to update and test your clinical cardiology diagnostic skills.



To cite Otto CM. Heart 2016;102:409-410.

Heart 2016;102:409-410.

doi:10.1136/heartjnl-2016-309463 